\title{
A Qualitative and Quantitative study of the Surgical and Rehabilitation Response to the Earthquake in Haiti, January 2010
}

\author{
A D Redmond ${ }^{1}$, S Mardel, B Taithe ${ }^{1}, \mathrm{~T} \mathrm{Calvot}^{2}, \mathrm{~J} \mathrm{Gosney}^{2}$, A Duttine $^{2}, \mathrm{~S} \mathrm{Girois}^{2}$
}

1. Humanitarian and Conflict Response Institute, University of Manchester, UK

2. Handicap International, France

Correspondence:

A. D. Redmond

Humanitarian and Conflict Response Institute, University of Manchester, UK, tony.redmond@manchester.ac.uk

Keywords: disaster, medical disaster response, humanitarian, earthquake response, Hiati

Abbreviations:

HCRI:Humanitarian and Conflict Response Institute

HI:Handicap International

INGO:international non-government organization

NGO:non-government organization

P\&O:prosthetic and orthotic

WHO:World Health Organization

doi:10.1017/S1049023X1100XXXX

\section{Abstract}

Background: The disaster response environment in Haiti following the 2010 earthquake represented a complex healthcare challenge. This study was designed to identify challenges during the Haiti disaster response.

Methods: Qualitative and quantitative study of injured patients carried out six months after the January 2010 earthquake in Haiti to review the surgical inputs of foreign medical teams.

Results: Study findings revealed a need during the response for improved medical records and data gathering for regulation, quality assurance, coordination and resource allocation; wider adherence to standard patient referral mechanisms and protocols linking surgical service provision with appropriate hospital and community based rehabilitation services; a greater recognition of the impact of non-amputation injury, and the need for patients to have a greater say in their management and to be the keepers of their medical records. Key first steps to improving the international response are a minimum dataset and uniform reporting.

Conclusion: This study showed that challenges for emergency medical response during the Haiti Earthquake involved issues of accountability, professional ethics, standards-ofcare, unmet needs, patient agency and expected outcomes for patients in such settings:

\section{Background}

On January 12, 2010 an earthquake of Richter magnitude 7.0 occurred off the coast of Haiti and near to its capital of Port au Prince. The Haitian earthquake affected an economically weakened country with critical losses of individuals and services. Also affected were government ministries, UN organizations, health services, transport, and aid agencies already present in Haiti. The number of injured has been estimated at perhaps 300,000, with 4,000 amputations reported to have been performed. ${ }^{1}$ At the time of the earthquake Haiti had a large non-government organization (NGO) presence and hosted a UN peacekeeping force. This presence enabled some international groups to be active early in the disaster response phase. ${ }^{2}$ The nature of the event, however, was such that many of the early response participants were initially disorganized and had to improvise to respond. A large range of medical response practitioners subsequently flew into Haiti.

The nature and scale of the international response has been documented and Haiti was overwhelmed and unable to control or contain the flow of this aid. Some observers of the early days of the Haiti disaster relief denounced some of the less reputable interventions as 'medical tourism. ${ }^{3}$ While there are reports of abuses, this evidence should be put into the perspective of a largely successful intervention during which reports of malpractice has remained anecdotal. Many positive but fragmented interventions also took place. Unfortunately, it is impossible to capture the data for these interventions beyond the referral and postoperative records established by specialized medical relief providers such as Handicap International (HI). Data from HI and its partners have provided an opportunity to crosscheck some disaster response surgical data and has given an insight into rehabilitation efforts during the Haitian earthquake response.

This study was designed to provide information about surgical and non-surgical interventions that occurred in the medical response to the earthquake using both quantitative and qualitative approaches. The study objective was to describe potential ways to improve the medical response to earthquake-related injury and support the work of humanitarian responders with refinement of best practice policies and procedures. 


\section{Methods}

This was a joint $\mathrm{HI}$ and Humanitarian and Conflict Response Institute (HCRI) study funded by the UK Department for International Development (DFID). The study consisted of qualitative interviews and quantitative data collection six months after the earthquake event. Patients were interviewed using semi-structured narrative techniques that allowed for expansion on areas of interest. Interview prompts included questions relating to the services received, the way those replying during interviews were medically treated and the circumstances of that treatment.

Patients receiving surgery after the January 2010 earthquake in Haiti and up until 3 months after were included in the study. Surgery in this study was defined as a procedure requiring local or general anesthetic or sedation. Limb surgery was defined as any surgical procedure on a limb including amputation, debridement, fasciotomy, wound excision, irrigation, and change of dressing that required general regional or local anesthetic or sedation.

Rehabilitation was defined as the different means involved in functional recovery and community reintegration. Rehabilitation data was collected up to November 2010, a longer period than that for the inclusion criteria for surgery. In this study, rehabilitation included physical and occupational therapy and orthopaedic services as well as prosthetic and orthotic services and mobility-assistive devices.

For the interview part of this study, the following were key study questions:

1. What earthquake-related injury/complications presented over time for surgical evaluation?

2. What surgical or non-surgical interventions were provided?

3. What organizations were providing these services and what was their on-the-ground skill set?

4. What was the nature and quality of amputations for prosthetic fitting?

5. What earthquake-related post-injury/post-surgery rehabilitation needs presented over time for evaluation by rehabilitation professionals?

6. What rehabilitation services, including prosthetics and orthotics $(\mathrm{P} \& \mathrm{O})$, were provided over time for injured persons and new amputees?

7. What was the impact of surgical and rehabilitation services on the functional outcomes of people injured by the earthquake.

The study was reviewed and received Ethics Committee approval from the Haitian Ministry of Health.

Quantitative data was collected from organizations known to have provided surgical services during response to the earthquake. These organizations were invited to contribute anonymous data on surgical procedures performed. If data were insufficiently detailed for the study, organizations were invited to contribute summaries or breakdown of surgical activity as well as their published datasets. The HI/Christian Blind Mission $(\mathrm{CBM}) /$ Secretairerie d'Etat a l'Integration des Personnes Handicapees SEIPH database and P\&O center registers were also used for comparison of quantitative data, which included information on hospital, community, prosthetic-orthotic, and home based rehabilitation services. Statistics on rehabilitation were not collected across different organizations like those for surgery. However HI and CBM were two of the largest providers of rehabilitation services.

Where data sharing or availability was limited, a priority was to capture surgical procedures on a timeline following the earthquake, including date of procedure and type of procedure (e.g. debridement, irrigation, amputation (type and level), first or follow up procedure).

When feasible the following data was included:

- age and sex of the patient

- cause of the injury: earthquake or other trauma or other pathology requiring surgery

- type of anaesthetic

- location of the center where surgery was performed.

Similar data from more than one source was aggregated and entered into the study. Data was collected such that it could not be attributed to individual organizations or location. Anonymity was assured for participating agencies because data sharing is uncommon among agencies and can provoke concerns over how their organization and its activities might be compared with other providers.

Study Interviews were carried out and recorded by a female Haitian researcher in July-August 2010 using a HI/HCRI format with semi structured interviewing techniques ${ }^{2}$. This technique of interviewing starts from a simple set of questions and allows the patient to digress and expand. Supervision of interviews was provided by a $\mathrm{HI}$ field research coordinator. These interviews took place on $\mathrm{HI}$ premises after patients had given their consent to be interviewed. Interview responses were recorded in quiet and unthreatening surroundings. The interviews were recorded on site in Creole and translated into English in Manchester.

\section{Results}

A total of eight major surgical service providers in Haiti following the earthquake were contacted for inclusion in the study. All responded and four agreed to be included. Two were closely related and submitted one consolidated dataset. Separate data from four published datasets were also reviewed. In total, data from seven surgical providers were used in the study analysis. The analysis does not include surgical data from small providers or Haitian providers. The HI/CBM/SEIPH rehabilitation dataset contained information from nine community focal points, or antennas, eighteen hospitals and a domiciliary outreach team. Further data specific to $\mathrm{P} \& \mathrm{O}$ services were included from the HI P\&O Rehabilitation Center. Information on more than twelve thousand beneficiaries over the period January -November 2010 was collected. Data from other P\&O providers was not included.

There were no common standards, forms or instructions for recording diagnoses and procedures across the seven providers included in the study. Several had developed internal medical records, which varied in content and detail. For example, one set of medical records reflected data on the volume of medical and surgical activity, irrespective of the specific intervention. Two sets included medical and surgical history of procedures prior to consultation as well as detail of wound complications and failed procedures. Another provided comprehensive epidemiological data on injury causation, operative and anesthetic procedures, and details of staff involved in the procedures. 


\begin{tabular}{|c|c|c|c|c|}
\hline 1 provider & 2 providers & 3 providers & 4 providers & 5 providers \\
\hline 13 & 16 & 19 & 4 & 6 \\
\hline Self referrals & \multicolumn{2}{l|}{ Provider to provider referrals } \\
\hline $\begin{array}{l}\text { 18 patients, 48 referrals recorded } \\
\text { (highest 5 lowest 1) }\end{array}$ & $\begin{array}{l}22 \text { patients, 55 referrals } \\
\text { recorded (highest 5 lowest 1) }\end{array}$ \\
\hline
\end{tabular}

Redmond (c) 2011 Prehospital and Disaster Medicine

Table1- Distribution of referrals among interviewees

The aggregated data from the seven surgical provider organizations included 10,643 reported surgical procedures, including 1,476 amputations. (Table 1) This implies an average amputation rate per surgical procedure of $14 \%$ (range $<1-46 \%$ ).

Information on the timing of amputations was derived from medical records and reports from the seven surgical providers, patient reports from rehabilitation intake interviews (HI/CBM/ SEIPH dataset), and patient recall interviews 6 months following the earthquake.

Data from patient intake interviews regarding the timing of lower limb amputations in the 30 days following the earthquake were drawn from the HI/CBM/SEIPH dataset. These data indicate an increase in amputations from day one post-earthquake (six patient reports) to a peak on day five (twenty-four patient reports) with a rapid taper to five or less patient reports in all subsequent days after day nine. The same dataset indicates that in subsequent months the primary amputation rates remained low.

The qualitative data, which represented interviews of 82 patients receiving amputation rehabilitation services at six months following the earthquake reported a similar peak in amputations around days four to seven. The issue of consent relating to medical procedures was always asked but did not always elicit concise answers. The disturbed political circumstances of Haiti in the summer of 2010 disrupted the data collection and limited the sample size. Nevertheless the interview sample is representative of the study population in terms of age groups, diversity of circumstances, and range of views. One interview had to be interrupted when it became clear that recalling the earthquake event caused emotional distress. . The sample represented the age pyramid of the Haitian population. The gender balance was biased towards female respondents (60\%); this is assumed to be due to the data collector being female and within the treatment centers better known to women. The quality of the data collected from female participants tended to be superior in terms of details.

\section{Types of amputations and other presenting conditions} Amputation Revisions

Data from the HI/CBM/SEIPH database indicate that overall in the entire year $96 \%$ of amputations were primary procedures and $4 \%$ were revisions. Within the first month only 4 cases were revisions (less than 2\%).

Data from the surgical providers' datasets vary in detail and specificity regarding the types of amputations, including revisions. One provider organization reported $37 \%$ of patients presenting for amputation were actually amputation revisions for procedures done elsewhere.

Upper and lower limb amputations:

Only two of the seven provider datasets analyzed for the study reported upper limb amputations. Upper limb amputations reported by both of these providers accounted for about $1 / 3$ of the total amputations recorded.

A large surgery provider provided aggregated data for the first two weeks (13-28 Jan 2011) on upper and lower limb amputation with the limitation that revision or repeat procedures were included in the data. Of a total 1,081 amputations, 388 (36\%) were upper limb amputations and 693 (64\%) were lower limb amputations. The same provider reported treating 5,714 fractures of which 2,043 (36\%) were upper limb and 3,671 (64\%) were lower limb.

A small NGO surgical team collected injury breakdown by body region from one surgeon whose team included hand surgery expertise as part of their specialized plastic and reconstructive surgical ability with reported frequencies of hand 7 (20\%), arm 5 (14\%), and leg 23 (66\%). According to the rehabilitation center register, most (51\%) of the lower limb amputations were below knee amputations (BKA)(Figure 5).

\section{Presenting conditions \\ Compartment syndrome}

Compartment syndrome was recorded as the surgical condition in twenty-eight cases (1.5\%) among 1,858 surgical interventions performed by one hospital of the largest health provider of our sample.

\section{Tetanus}

One provider recorded ten cases of tetanus from 13 Jan to 2 May, with nine of these occurring in the first two weeks after the earthquake. Another reported four cases and the University Hospital of Haiti reported one case.

\section{Crush syndrome}

Crush syndrome was not recorded by the seven organizations included in this study. One organization published elsewhere that their specialist renal team reported nineteen patients with crush syndrome referred for haemodialysis ${ }^{4}$. They concluded that the low number was probably related to an overwhelming number of severely injured people who died early on; survivors who were pulled from the rubble early by bystanders who were not crushed for long periods; and many who could not receive timely medical care.

Rebabilitation of amputees and community based services

The rehabilitation dataset was recorded from Jan to November 2011 to allow for a greater reliability and time for data collection. Rehabilitation statistics from the HI/CBM/SEIPH database showed that the rehabilitation teams at the $\mathrm{P} \& \mathrm{O}$ center had registered 424 amputees in the period February-November 2010 and 698 amputees across the whole range of rehabilitation services. The center provided lower limb prostheses only during this period. Of this amount, 283 (66.7\%) were recorded as having been a direct result of the earthquake.

Of the 141 non-earthquake related amputees attending the center between February and November, $84 \%$ had their amputation before the earthquake and presented for either new or replacement prosthetic fitting or because their previous prosthesis had been lost or damaged as a result of the earthquake. The fourteen post-earthquake amputations were for a variety of reasons including both trauma (e.g. road traffic accidents) and medical (e.g. diabetic ulcer).

The number of amputees seen by HI rehabilitation teams is outweighed by the number of beneficiaries receiving rehabilitation for 
other injuries. Amputee rehabilitation accounted for 3.5\% to $6 \%$ of the total demand for services in terms of number of beneficiaries. $3.5 \%$ is the total proportion of people assessed for prosthesis. $6 \%$ is the total number of registered amputees across all rehabilitation services, which include upper limb amputees and those not ready for prosthesis. More rehabilitation sessions for clients took place in community outreach initiatives with rehabilitation capacity than at institutional based rehabilitation settings. Between February and November 2010 the number of orthotic and number of prosthetic patients seen at the $\mathrm{P} \& \mathrm{O}$ center was relatively equal: 424 to 364 .

Limiting the validity of the study was that the data for 1,476 amputations was from larger surgical providers that represented only seven major organizations and did not include Haitian providers. The study is likely to include some double counting as several providers did not distinguish primary amputation from repeat procedures. Few agencies distinguished the cause of injury (earthquake or non-earthquake related). Some amputations performed post-earthquake were not as a direct result of the earthquake (24 of a total of 45 amputations by one agency or 53\%). Rehabilitation statistics for amputee patients show earthquake related amputations were predominant in the first few months with a steady increase in the number of non-earthquake related assessments in the later period. The need for prosthetics as a measure of amputation will underestimate the total number of amputees as upper extremity amputees are not usually provided with prosthesis in these circumstances. The proportion of upper extremity amputation may be about $33 \%$ of the total. Amputees who failed to survive may not have been included, and one report suggests even hospital deaths might not have been recorded due to absence of initial note keeping. Amputation of digits and thumb are seldom included in datasets but can represent a very disabling disability. Amputations accounted for only a small percentage of conditions presenting for rehabilitation services.

The interview data contradict some of the quantitative findings by suggesting that fewer amputations may have taken place in the first few days of the event than was thought. This may reflect a potential confusion between patient understanding of "first procedure" and "amputation". If correct it would indicate that most amputations were carried out by incoming teams rather than those already on site. While delays were common between the earthquake and the amputation, these delays were not often attributable by the patients to conservative practices but rather to congestion and neglect. This was consistent with previously published reports that described most patients not being attended to by medical staff until infection had become obvious, even to friends and family. 4

The interviewer asked as precisely as possible how the patients related to the medical services they encountered. The answers tended to be fairly general as to the identity of the providers (Haitian or foreign) but precise in terms of familiar health providers naming sites of hospitals or field hospitals. The choice of one or another site for first treatment seems to have been determined by convenience and proximity. Patients were sometimes removed late from the rubble because family was not close and could not help. Many patients reflected on the paucity of treatment available prior to the earthquake and for some with preexisting conditions on the cost of medical services in Haiti. Some noted that on day three they were asked by their health providers to provide clean cloth to cover wounds. The intervention of foreigners is sometimes perceived to have led to examinations but no follow-up and loss of records. The perceived medical hierarchy between Haitians and foreigners and different groups of foreigners can be sensed in the interviews with some emphasis on "eminent specialists" which has been noted in other studies of foreign interventions in Haiti prior to the earthquake. ${ }^{5}$

Patients mentioned informed consent more frequently when referring to late amputations and the process of consent was often associated with a positive appreciation of treatment. The issue of consent was tacitly or tersely acknowledged in most interviews and it was more fully discussed in very few cases. When it is discussed more fully it was to show that the patient took control of the situation. For example, one patient claims to have gone 'on strike' after eight 'operations'. Yet the same patient emphasized that the treatment was ultimately good because it could be measured in terms of a large volume of medication.

Comments on treatment available immediately after the quake were often more critical and showed the importance of local helpers (cleansing by a private doctor). The fear of amputation was present on day two of the emergency with rumor a possible factor worthy of further study. Some patients refused to enter a hospital because of rumor of amputation. For example, a patient with a broken foot left the hospital untreated with the fractures healing spontaneously but then requiring therapy for two months. Only a few patients mentioned rejecting an amputation outright; in a few cases a relative refused the amputation of a dependent.

While the fear of amputation appeared widespread there was an equal awareness of the concept of infection. Many patients displayed a good understanding of life threatening nature of crushed limbs.

Patients showed a good understanding of the role of external fixators. Occasionally patients asked to have their limb removed after a long and painful battle with infection. For some patients the earthquake aggravated a pre-existing ailment rather than creating the condition. Some related the earthquake with a worsening of their chronic condition as in increased blood pressure.

Some patients migrated through several hospitals, even patients with considerable wounds or the loss of limbs, and experienced a number of new hospital encounters with establishment of new relationships and triggering new medical records. However patients did not own their medical record and in the first few weeks the referral process was often not documented. This suggests that data sets provided by medical providers will to some extent replicate patient records, potentially inflating the numbers of patients and possibly exaggerating their conditions.

The number of self-referrals was almost equal to that of health-provider to health-provider referrals. When patients were immobile, their families sometimes enabled the move. Some patients noted that they heard of therapeutic centers by radio, but none made reference to the advances in communication technology which were supposed to have been central in the response to the Haiti earthquake.6 Rumors and informal information networks seem to have helped patients make choices and often migrate from one provider to the next (Table 4). On average self-referring patients moved around 2.66 providers and when transferred by providers moved on average 2.5 times. This use of self-referrals reflected the situation of bottleneck which has been documented by others and which only eased several months after the crisis following the intervention of USS Comfort and large INGOs. 


\section{Issues determined from the qualitative (interview) data}

- There were a number of self-referrals and moves from one provider to another, as patients sought the best possible treatments measured according to consumer led indices. This implies that a number of patient strategies need to be considered in order to deliver aid most effectively.

A patient's seeking of health care was informed by rumor and only occasionally by the media or new technologies.

- Patients measured the quality of services by the measurable experience of the availability of medication or the frequency of visits.

- Amputations did not take place in the first few days of the emergency; with very few exceptions where the limb was already lost.

- While delays are common between the earthquake and amputations, these delays were attributed to congestion and neglect. (it is possible that conservative practices may have been regarded as under-treatment by the patients)

\section{Data Limitations}

Data for this study represented that from over ten thousand surgical procedures and over nine thousand beneficiaries of some form of subsequent rehabilitation or support; however there were limitations in our ability to compare data sets. Surgical data were taken from either published reports or supplied information, but being retrospective there is no means of validating the accuracy of the data. Missing data and variability restricted the amount of items that could be used for comparison and the lack of detailed medical records meant that little comment can be made on the appropriateness or quality of treatment. No data could be collected from small providers or Haitian providers, which seem to have been numerous and collectively may have undertaken a significant number of surgical procedures.

The rehabilitation database which contained the broadest available range of data on the breadth of disabling injuries including amputations was limited in terms of recording mechanisms due to the possibility of duplication of statistics between services (especially in the early post-earthquake period before a unique ID numbering system was established) and inconsistency of recording given human resource and managerial capacities.

Qualitative interview information collected in terms of a life story contains the potential for inconsistencies and relies on subjective opinion on issues and recall of specific issues occurring at a highly chaotic time.

\section{Discussion}

The lack of evidence of a sophisticated or coordinated registration process, despite requests by coordinating agencies to submit surgical workload and capacity was a significant finding in itself. It is estimated that within a month of the earthquake more than 600 organizations were providing emergency humanitarian aid to Haiti with 274 organizations conducting health activities, but the exact number of providers delivering surgical help is unknown ${ }^{6}$. Recording of statistics or even basic medical record keeping appears to have been given low priority, which has also been noted in other studies. ${ }^{7}$ This raises significant ethical concerns and deserves a comparison of surgeons' record keeping in domestic and overseas settings. However it should be also noted that this may not solely reflect poor practice of individuals or organizations, but also reflect the lack of pre-preparedness of the international response to standardize reporting and recording mechanisms.

The continuity of care was also negatively impacted by the lack of continuous and standard record keeping. Our findings showed that the HI/CBM/SEIPH database records "date of amputation" as 12 Jan 2010 (date of earthquake) in 68 out of $242(28 \%)$ cases of lower limb amputation. If correct, this would support a greater involvement of local services or NGOs already present than has been hitherto recognized. However qualitative data at interview did not support this, and the organization considered it likely that these patients were mistakenly reporting the amputation date as "date of injury", since they were collecting the information subjectively from clients rather than being presented with a medical record of their procedures.

While the surge of medical relief undoubtedly broadened the range of medical expertise it created a wild market rather than coordinated help. Qualitative findings suggest it is likely that the unchecked access to a diversity of medical providers was used strategically by patients to be selective for what they considered to be the best care. The significant proportion of self or family referrals in the rehabilitation data seems to support this. The rumors of amputation already present by day two may have contributed to this. Earthquake victims were therefore not the passive recipients of care some providers might have assumed. Cultural factors were at play in determining quality of care which was often measured by the patient by the volume of medication and the availability of X-ray facilities and medical staff. Similarly the fact that several people reported "shopping around" between several organizations for prostheses (though with less clear definitions on how quality was being perceived) suggests that many people may have had multiple prostheses manufactured. The use of traditional healers may be under recognized although two patients did make reference to seeking such help in the first instance.

The rate and timing of amputation carried out by surgical teams varied, and between some varied very significantly. In one surgical facility amputation was less than $1 \%$ of surgical procedures and yet in another it appeared to be over $45 \%$. The difference could reflect the different biases such as arrival post earthquake, case complexity, patient access, referrals and previous wound neglect. However the same magnitude of difference is not seen with other surgical teams already in the country and in theory being accessible to patients with similar degrees of severity, urgency or delay in presentation. There was also data from one organization suggesting relatively similar (high) amputation rates in both non-earthquake and earthquake related trauma suggesting there is a significant difference in practice between some national teams. At a more profound level it might reveal different approaches to the so-called 'surgeon's dilemma' or the balance between early amputation with less risk of infection and crush syndrome but greater disability and repeated limb salvage procedures to preserve limb length and reduce disability at the risk of later infection and crush syndrome and treating fewer individual patients.

The team with the lowest rate of amputation was a combined orthopedic and plastic surgery team which may be significant as this additional expertise can salvage some limbs which might otherwise have been non-viable due to a lack of soft tissue cover. It is worth noting that the long term functional outcome is not known for these patients. 


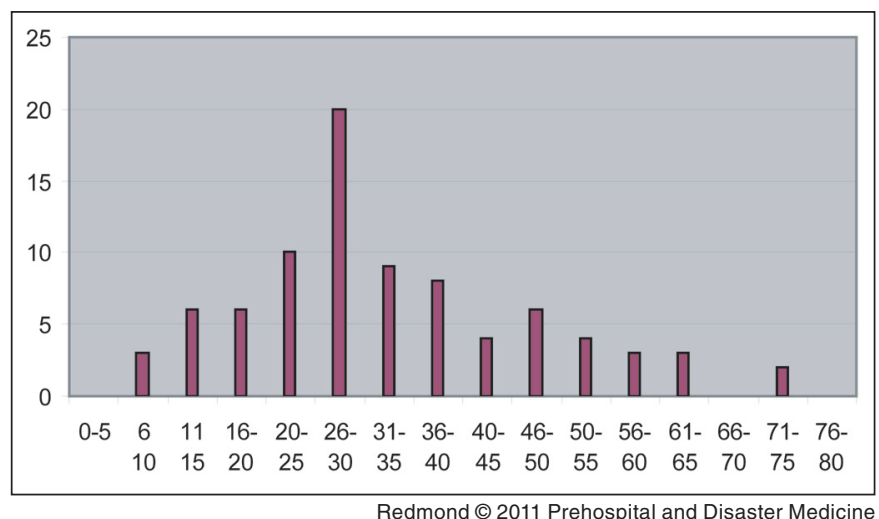

Figure 1-Age distribution of the sample interviewed in July-August 2011

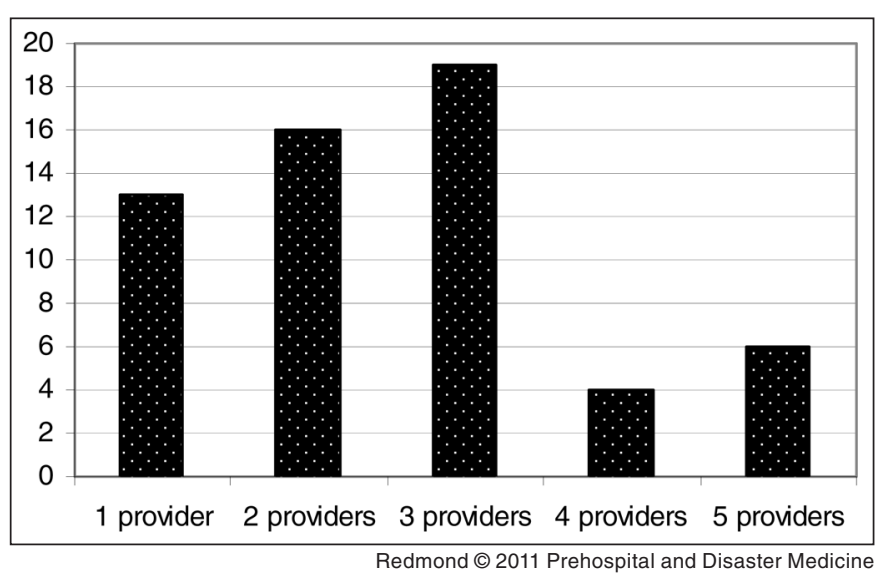

Figure 2-Breakdown of patients and sessions across rehabilitation settings

While the significant numbers of recordings of "date of amputation" as day one in the HI/CBM/SEIPH database seems to be a false finding, if correct it would indicate that most amputations were done by surgeons already in country at the time the earthquake occurred. Because working conditions, surgical need, wound complications and surgical availability are so dependent on the timing after earthquake, any comparisons of the procedures and amputation rates may be useful if constantly examined with respect to the timeline postearthquake as shown in the graphs in Figures 2, 3, 4 and cumulatively in figure 9. For example after the first two weeks surgical needs were estimated to be diminishing while surgical availability and specialist expertise had significantly increased. ${ }^{7}$ Based on the quantitative and qualitative findings, the amputation peak at day five seems most reliable, suggesting that most were performed by incoming teams rather than pre-existing ones.

Data from the rehabilitation database illustrate that amputation as a source of disability can be over represented when set alongside the consequences of other injuries. For example hand injuries and related subsequent significant disability appears largely overlooked. Also amputations of the digits including the thumb (which supports most of the function of the hand and can be a devastating disability), appear not to have always been counted, with the exception of organizations that had hand

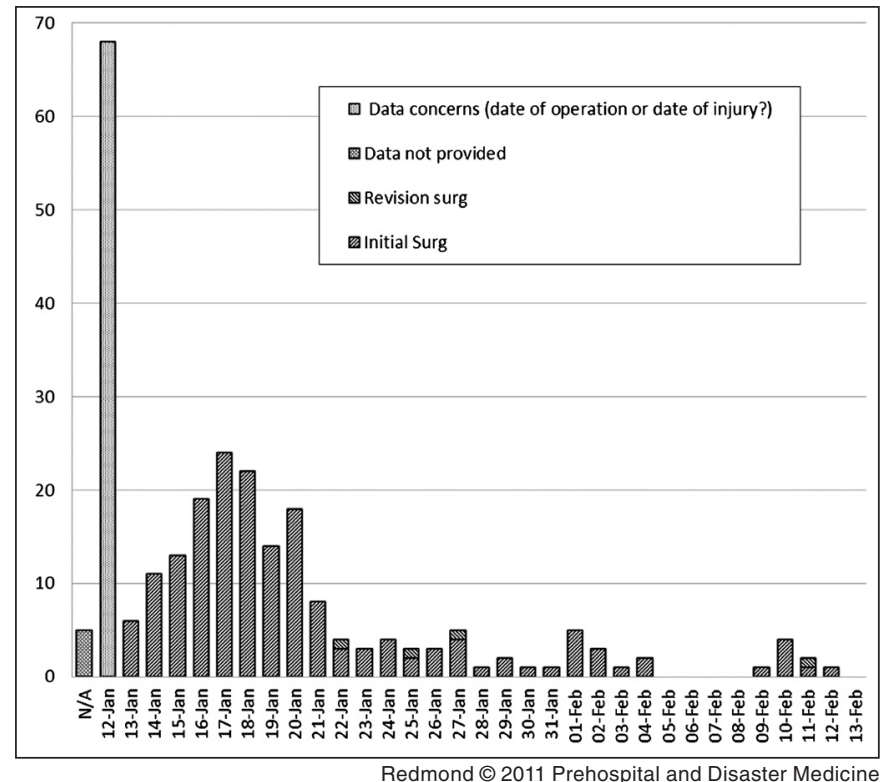

Figure 3-Amputations per day according to records made at Handicap International P\&O Rehabilitation Centre

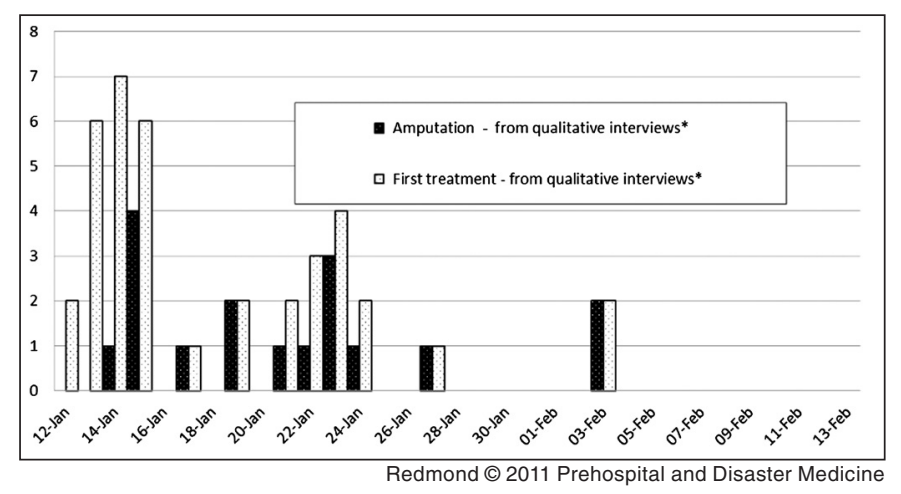

Figure 4-Timing of amputations according to patient recall 6 months following the earthquake

surgery expertise as part of their specialized plastic and reconstructive surgical ability.

Guillotine amputations as a primary and only procedure were carried out and were reported anecdotally, although the records are too poor to establish how many and in what circumstances. Published reports highlighted the controversy ${ }^{16}$ over different organizational approaches and some of the difficulties caused by them. The current surgical guidelines from WHO which are also incorporated into guidelines for disaster situations contain advice on the use of this surgical procedure which may not convey adequately the relatively infrequent conditions with which it is now indicated as a primary and sole procedure.

The initial rehabilitation response took place at the hospital level, closely followed by a community response. The presence of a rehabilitation team at the hospital level was key not only in initiating the early rehabilitation process and providing essential advice for the patient and carers, but also in pre-identifying conditions that will need longer-term rehabilitation follow-up at a community level. While more sessions per patient were provided at the hospital level (average of 15 sessions per patient), community level physical rehabilitation allows a longer-term follow-up 


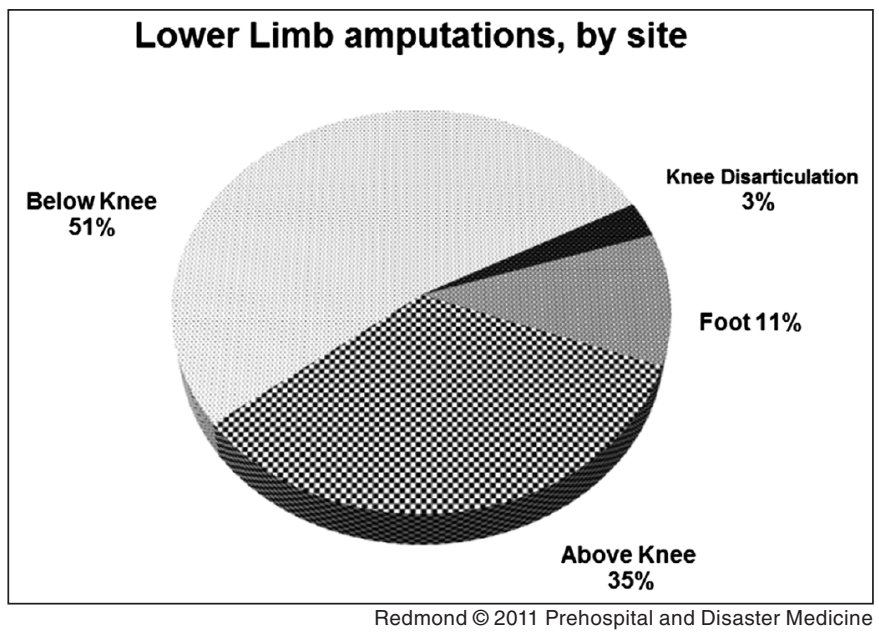

Figure 5-Lower limb amputations (EQ related only) over 6 months following the earthquake (Handicap International $\mathrm{P} \& \mathrm{O}$ Rehabilitation Centre)

and more importantly, a rehabilitation process that takes into account wider factors that may impact upon the outcome of the overall process, such as multidisciplinary approach involving psychosocial support, management of physical environment challenges and commitment of family and carers.

Initial findings also suggest a high turnover of patients in secondary structures in link with a very quick discharge process, which makes it essential to maintain mechanisms to link between secondary structures and community for continuity of treatment.

The fact that the majority of patients seen for rehabilitative services (hospital or community based) were not amputees, but were a mixture of injuries directly related to the earthquake, and other conditions and disabilities requiring rehabilitation services, has implications for future disaster planning. A greater emphasis was placed on rehabilitation work carried out at the secondary level providing rapid services for persons having sustained an amputation, including the early provision of prostheses. This may have been prompted by the heavy international media focus towards this area. The benefits of early fitment of a fresh amputee with a prosthetic device are well established, including a better preservation of muscular strength, joint flexibility and gait pattern that ease considerably the rehabilitation process afterwards. However, it appears that the need of rehabilitation for complex and multiple injuries, particularly involving the quick provision of orthotic devices for hand surgery or nerve damage injuries for example, was certainly greater and equally urgent. Indeed, from a rehabilitation perspective, the initial 3 months are crucial in preventing contractures and other complications and preserving flexibility and limbs function. The immediate availability of orthoses and mobility devices is crucial in this matter, while management of amputation does not necessarily imply swift provision of prostheses.

It would seem that very few of the medical \& surgical service providers came with orthotic provision capacity, despite the fact that they would be dealing with complex or multiple injuries, including peripheral nerve injuries which comprised a bulk of the need.

Furthermore the high number of amputees picked up through the community outreach initiatives suggests that direct referral routes were the exception rather than the rule (though some positive examples of direct collaboration between surgical and rehabilitation was evident). Again this could adversely affect the continuity of care and access to rapid rehabilitation follow up which could, in turn, impact upon the long-term functional outcomes of a patient or group of patients. In saying that, the success of the community based rehabilitation teams in both identifying community based clients and either referring or offering access to treatment in local settings seems to have been a positive strategy. Hospitals did refer to these settings as part of the rehabilitation strategy. This strategy needs to be further built on and formalized in future disasters to improve the efficiency of this referral process.

\section{Concluding Recommendations}

Our recommendations for future post-earthquake surgical responses draw on the study findings and the challenges they reveal around the response of foreign medical teams to a disaster such as Haiti. These challenges involve issues of accountability, professional ethics, standards of care, unmet needs, patient agency and expected outcomes for patients in such settings:

- Improved medical records and data gathering for regulation, quality assurance, coordination and resource allocation. We recommend a common dataset with minimum data requirements for all foreign medical teams. These data should be shared with the host country, coordinating agencies and among provider organizations.

- Wider adherence to standard patient referral mechanisms and protocols linking surgical service provision with appropriate hospital- and community-based rehabilitation services. Ideally, a comprehensive health response following an earthquake will equate successful outcomes with prevention of long-term disability and not simply lives saved. The registration of foreign medical teams providing surgery and rehabilitation in such situations would provide an opportunity for more effective inter-provider coordination $^{8}$.

- Anticipation of injuries to the upper limb and those not requiring amputation. Specialized training to registered foreign medical and rehabilitation responders should include skills for the management of complex fractures, injury to the hand, prescription and use of orthoses and other assistive technologies.

- Relevant medical information at the point of care: keep the medical record with the patient. In Haiti following the earthquake, the patient was the only common point between all stakeholders involved in their care. We recommend that for future post-earthquake foreign medical response, all essential information on every medical/ surgical procedure and related recommendation for follow-up should be documented on pre-translated forms and handed to the patient to assist future assessment, consent, theatre, wound care, post-operative, discharge and rehabilitation planning.

- Early identification of influences on patients' careseeking behavior and preferences. This study revealed significant patient choice of care provider, self-referral to multiple providers, acceptance or not of surgical intervention following the earthquake in Haiti. Influences on 
these choices can have both positive and negative impacts on patient outcomes. Foreign medical teams should not underestimate the extent to which social networks

\section{References}

1. PAHO/WHO Situation Report on Health Activities Post Earthquake Sit Reps 2 March 2010, 18 May 2010 http://www.docstoc.com/docs/43990290/Eitel-HaitiFeb-Mar-2010-Report--final-draft\# accessed 14/11/11.

2. Barriball KL While A :Collecting data using a semi-structured interview: a discussion paper,.J Adv Nursing1994;19(2):328-335.

3. Earthquake in Haiti one year later, January 2011, PAHO/WHO, 2011, pp. 6-8. http://new.paho.org/disasters/index.php?option=com_content\&task=view\&id=14 $75 \&$ Itemid $=1$ accessed 14/11/11.

4. Van Hoving DJ, Wallis LA, Docrat F, De Vries S: Haiti disaster tourism-A medical shame. Prehosp Disaster Med 2010;25(3):201-202.

5. Vanholder R, Gibney N. Luyckx VA, Sever MS Renal disaster relief task force in Haiti earthquake. Lancet 2010;375(9721):1162-63. influence contact with service providers. We therefore recommend early dialogue with patients and community leaders to detect where such influence plays a role.

6. Brodwin PE "Politics, Practical logic and Primary health care in rural Haiti" Medical Anthropology Qauaterly 1997;11:1,69-88.

7. PAHO/WHO January 24, 2010 Emergency Operations Center Situation Report Number 11, Haiti Earthquake). http://reliefweb.int/node/341573 accessed 14/11/11.

8. Paranteau WHHavens JM Harrington S Gates JD (2010) "Reestablishing surgical care at Port au Prince general hospital Haiti”J AM Coll Surg 211,1,126-130.

9. Redmond AD, O’Dempsey TJ, Taithe B “ Disasters and a register for foreign medial teams” Lancet 2011; 377(9771):1054-55. 